\title{
Editorial
}

\section{Vascular Surgery: Growing with Diversification}

\author{
Sanjay C. Desai ${ }^{1}$ \\ ${ }^{1}$ Department of Vascular and Endovascular Surgery, MS Ramaiah \\ Medical College and Hospital, Bengaluru, Karnataka, India
}

Int J Recent Surg Med Sci 2019;5:36

The art of surgery has evolved since the documented time of Shushrutha and is growing since then. The growth has been fast and huge. In the growing trend, minimal access surgeries became popular, and, in the recent times, percutaneous interventions have become surgical alternative when and where possible.

Vascular surgery is amongst the youngest subspecialty to grow in the field of surgery and has been evolving rapidly. It involves the treatment of disorders of veins, arteries, and lymphatics. The thoracic and cranial vascular disorders were left out of vascular surgery, as it was already saturated with its own progress. The current vascular surgeon is a physician, surgeon, and interventionist, as he covers medical management, surgical reconstruction, and endovascular intervention in providing complete care for vascular disorders.

The growing burden of diseases has been increasing, and the growth of surgery is still continuing to catch up. Vascular surgery is no exception and is continuing to evolve and diversify in providing the best care for vascular diseases.

The common arterial disorders managed by a vascular surgeon include Buerger's disease, atherosclerotic peripheral diseases, diabetic foot and angiopathy, arteritis, acute thrombosis and embolism, and traumatic injuries. Varicose vein and its complications make the most of venous disorders treated. The incidence of venousthromboembolism, deep vein thrombosis (DVT), and pulmonary embolism is increasing with a rapid rate. Primary and secondary lymphedema and other lymphatic disorders are being treated by vascular surgeon but still a lot more needs to be done in this field.

Vascular surgery is a recognized subspeciality in most of the practices across the world. In India, both the Medical
Council of India and the Diplomate of the National Board have recognized vascular and endovascular surgery as an independent specialty. There are a total of 37 seats of MCh and DNB vascular surgery being offered across the country annually.

The management of peripheral vascular diseases has evolved, such that effective treatments can often be offered with minimal hospital stay and as day care procedures in many cases. Vascular surgery has evolved such that the effectiveness of a procedure or treatment is critically evaluated in clinical research studies in thousands of patients and measured by single-digit percentages. The pathophysiology and genetic basis of vascular diseases are now understood so well in some cases that disease processes are managed effectively with nonoperative means. The rapidity with which the treatment of vascular diseases has evolved over the past century is remarkable. We can only imagine how the practice of vascular surgery will look during the next 50 years, if such great progress continues.

It is my privilege to offer you this edition of the International Journal of Recent Surgical and Medical Sciences dedicated to vascular surgery. We have tried to cover various topics of interest submitted by young vascular surgeons. We hope this issue would give our readers a fresh perspective on vascular diseases and help young surgeons in growing and diversifying into a vascular surgeon.

Note

S.C.D acts as the co-editor of the current issue.

Conflict of Interest

None declared.

\footnotetext{
Address for correspondence Sanjay Desai, MS, Department of Vascular and Endovascular Surgery, MS Ramaiah Medical College and Hospital, Bengaluru 560054, Karnataka, India (e-mail: scdesai@hotmail.com).
}

DOI https://doi.org/ 10.1055/s-0039-3401467 ISSN 2455-7420.
(C)2019 Medical and Surgical Update Society
License terms

(요 (1) $\Theta \circledast$ 